\title{
Effect of integrated weed management practices on performance of dry direct seeded rice (Oryza sativa $\mathbf{L}$.)
}

\author{
KP Bhurer ${ }^{1}$, DN Yadav ${ }^{2}$, JK Ladha ${ }^{3}$, RB Thapa ${ }^{2}$ and K Pandey ${ }^{2}$ \\ ${ }^{1}$ Nepal Agriculture Research Council (NARC), ${ }^{2}$ Institute of Agriculture and Animal Sciences \\ (IAAS), Nepal, and ${ }^{3}$ International Rice research institute (IRRI), the Philippines
}

\begin{abstract}
Weeds are serious problem in dry direct seeded rice (DDSR). A field experiment was conducted during rainy seasons of 2010 and 2011 at research farm of the Regional Agricultural Research Station (RARS) Parwanipur, to study the effect of integrated weed management practices on the performance of dry direct seeded rice. Ten treatment combinations viz; weedy, weed free (weekly), Pendimethalin $\mathrm{fb}$ (followed by) Bispyribac, Pendimethalin $\mathrm{fb}$ two hand weeding, Stale seedbed $\mathrm{fb}$ Bispyribac, Stale seedbed fb Pendimethalin fb Bispyribac, Mulch $4 \mathrm{t} / \mathrm{ha} \mathrm{fb}$ Bispyribac fb one hand weeding, Stale seedbed $\mathrm{fb}$ mulch $4 \mathrm{t} / \mathrm{ha} \mathrm{fb}$ Bispyribac, Pendimethalin and Sesbania co-culture $\mathrm{fb}$ 2,4-D Na salt $\mathrm{fb}$ one hand weeding and Pendimethalin $\mathrm{fb}$ 2,4-D fb one hand weeding were tested in a randomized complete block design and replicated thrice. Observations were taken on weed, plant growth and yield attributes, yield, and socio-economic parameters. All weed control treatments significantly reduced the weed density and dry weight of weed resulting significant increase in yield of DSR over weedy check in both years. Weed free treatment resulted the highest yield, however, it was not economical due to high cost of cultivation. The use of Pendimethalin $\mathrm{fb}$ 2,4-D fb one hand weeding produced yield (5161 in 2010 and $6160 \mathrm{~kg} / \mathrm{ha}$ in 2011) which were statistically at par with yield (5305 in 2010 and $6319 \mathrm{~kg} / \mathrm{ha}$ in 2011) obtained under the weed free treatment. Further, the highest benefit cost ratio (CBR) 1.77 and 2.22 and net return Rs 47700 and 75084/ha during 2010 and 2011, respectively, were obtained under this treatment indicating its superiority over other treatments. The grain yield, yield attributing characters viz. panicles per $\mathrm{m}^{2}$, panicle weight, filled grain per panicle, thousand grain weight as influenced by different weed management practices revealed that higher yield resulted from weed free plot followed by Pendimethalin followed by two hand weeding and Pendimethalin followed by 2,4-D followed by one hand weeding. However, the net return per unit investment resulted highest in Pendimethalin followed by 2,4-D followed by one hand weeding. This proved that amid increasing wage rate and labor scarcity integrated weed management through Pendimethalin 30 EC (stomp) @ $1 \mathrm{~kg}$ a. i./ha as pre- emergence herbicide application followed by 2,4-D sodium salt 80 WP @ $0.5 \mathrm{~kg}$ a.i./ha followed by one hand weeding or stale seed bed followed by Pendimethalin 30 EC (stomp) @ $1 \mathrm{~kg}$ a. i./ha followed by Bispyribac (nominee gold) @ $25 \mathrm{~g}$ a. i./ha $10 \%$ @ $200 \mathrm{ml} / \mathrm{ha}$ at 20 days of seeding resulted best alternative for manual hand weeding practices giving higher net return per unit investment.
\end{abstract}

Key Words : direct-seeded, weed, herbicide, rice, yield

\section{Introduction}

Rice is the main staple food of Nepalese People and it is predominantly grown by transplanting in puddled soil with continuous flooding. However, this method requires huge amount of water, labor and energy for land preparation, nursery raising, transplanting and weeding leading to high cost of cultivation. Furthermore, puddling also affects soil health due to the dispersion of soil particles, soil 
becoming compact and making tillage operations difficult requiring more energy in succeeding crops such as wheat (Singh et al., 2002 \& Hobbs et al., 2002). An alternative to puddling and transplanting could be aerobic direct seeding because it requires less water, labor and capital inputs. The direct-seeded crop also matures earlier (7-10 days) than the transplanted crop, thus allowing timely planting of the succeeding wheat crop (Giri, 1988).

Irrigated "aerobic rice" is a new system being developed for low land areas with water shortage and for favourable upland areas with access to supplementary irrigation (Tuong et al., 2003 and Belder et al., 2005). However, aerobic systems are subject to much higher weed pressure than conventional puddled transplanting systems (Rao et al., 2007). Aerobic soil dry-tillage and alternate wetting and drying conditions, on the other hand, are conducive to the germination and growth of weeds causing grain yield losses of 50-91\% (Elliot et al; 1984; Fujisaka et al. 1993 and Rao et al., 2007). Weed account for $50-80 \%$ yield reduction in rainfed uplands (Ranjit et al., 1989).

Thus, weeds are the most severe constraint to aerobic rice production and timely weed management is crucial to increasing the productivity of aerobic rice. Most upland and aerobic rice growers in Asia mechanically weed their crops two or three times per season, investing upto 190 person days $\mathrm{ha}^{-1}$ in hand weeding (Roder; 2001). Herbicides are considered to be an alternative/ supplement to hand weeding. Both pre-emergence and post-emergence herbicides can be used in aerobic rice fields and they are effective, if properly used (De Datta et al., 1996 and Singh et al., 2006).

Since the concept of aerobic rice is new growing rice under aerobic conditions on raised beds or on flat land would require suitable, effective and economic weed-control methods, development of new improved herbicides for aerobic dry-seeded rice is also needed. The increasing cost of labour threatens the sustainability of transplanted rice within the rice-wheat system of Indo-Gangetic Plains. Direct-seeding is cost effective, can save water through either rice crop establishment and allows early sowing of wheat (Ladha et al., 2003 and Singh et al., 2003). In RCT ${ }^{\mathrm{s}}$, dry-DSR with zero-till machine has been started and information on weeds and weed management is scarce. However, this type of research works are less carried in our context. Therefore, the present experiment was conducted to develop effective and economical integrated weed management practices for dry direct-seeded rice.

\section{Materials and methods}

The field experiment was conducted for two consecutive years (2010 and 2011) at the experimental field of RARS, Parwanipur at an elevation of $115 \mathrm{~m}\left(27^{\prime \prime} 4 ' \mathrm{~N}\right.$ and $\left.84^{\prime \prime} 53^{\prime} \mathrm{E}\right)$. The climate of Parwanipur is subtropical with very hot summers and cold winters. The hottest months are May and June, when the maximum temperature reaches $40^{\circ} \mathrm{C}$, whereas during December and January, the coldest month of the year, the minimum temperature often goes below $7^{0} \mathrm{C}$. The average rainfall is $1200 \mathrm{~mm}, 80 \%$ of which is received through the monsoon from June to September. The experimental soil was an Inceptisol formed on Himalayan residium with the following characteristics in the top $15 \mathrm{~cm}$ profile. clay $8.0 \%$, silt $17.0 \%$, and $75 \%$ (loamy sand), $\mathrm{pH}$ (1.2 soil. water) 7.0 total $\mathrm{N} 0.086 \mathrm{~g} \mathrm{~kg}^{-1}$, total C $6.5 \mathrm{~g} \mathrm{~kg}^{-1}, \mathrm{NH}_{4} \mathrm{OAC}$-extractable $\mathrm{K} 0.054 \mathrm{~g} \mathrm{~kg}^{-1}$, Olsen $\mathrm{P}$ $0.015 \mathrm{~g} \mathrm{~kg}^{-1}$, Saturation extract exchangeable cations $1.4 \mathrm{ds} \mathrm{m}^{-1}$, and bulk density $1.6 \mathrm{Mg} \mathrm{m}^{-3}$. 
The experiment was arranged in randomized block design with three replications. Ten treatments were weedy check, weed free (weekly hand weeding), Pendimethalin followed by Bispyribac, Pendimethalin followed by two hand weedings, stale seedbed followed by Bispyribac, stale seedbed followed by Pendimethalin followed by Bispyribac, mulch (wheat straw) @ 4t/ha followed by Bispyribac, Pendimethalin and Sesbania co-culture followed by 2,4-D Na salt followed by one hand weeding stale seedbed bed followed by mulch (Wheat Straw) $4 \mathrm{t} / \mathrm{ha}$ followed by Bispyribac and Pendimethalin followed by 2,4-D followed by one hand weeding. Herbicides were applied using a power operated Knapsack sprayer with a flat fan nozzle and water as a carrier at 750 liter ha ${ }^{1}$. For the weed free treatment, 8-11 hand weeding were done to maintain a weed free situation. In the weedy control, no weeding was done. Sabitri, a late duration variety was seeded on $7^{\text {th }}$ July, 2010 and $15^{\text {th }}$ June, 2011 with seed rate of $30 \mathrm{~kg} / \mathrm{ha}$ by Pantanagar Zero seed drill in line. Row to row spacing was kept $20 \mathrm{~cm}$ and plant to plant continuous. Pre-sowing irrigation was applied in all the plots for moisture. In stale seedbed treatment, the pre-seeding herbicide Glyphosate @ $1.0 \mathrm{~kg}$ a.i/ha was applied 10 days before seeding to kill already germinated weeds. In Sesbania co-culture treatment, it was sown as an inter crop with rice.

\section{Results and discussion}

\section{Effect on weeds}

The major weeds infesting the experimental field were Cynodon dactylon, Cyperus rotundus, Cyperus iria, Echinochloa crusgalli, Echinochloa colona, Fimbristylis dichotoma, Palantus nirui,etc. However, the number of weeds infested the experimental field were less in 2011 than 2010, perhaps due to more rainfall in 2011 which might have suppressed the growth of some weed species. All the weed control treatments significantly reduced the weed density and dry weight of weeds over weedy check during both the year of study. The maximum weed population and weed dry weight were recorded in weedy check during both the years. Among the herbicidal treatments maximum intensity and dry weight of weeds were recorded in Pendimethalin fb by Bispyribac and the lowest intensity and dry weight of weeds were recorded in Pendimethalin fb 2,4- D fb one hand weeding. The next lowest intensity and dry weight of weeds were recorded in Pendimethalin fb two hand weeding. This indicates that one cannot rely on herbicide only for the weed control and at least one hand weeding is needed. During both years, the proportion of grassy weed dry weight was higher than other weeds (table- 1.a, 1.b, 2.a \& 2.b). Grasses persist in all of the principal crops and are a major cause for concern. It is also reported that the greatest weed pressure and crop-weed competition occur in aerobic rice and least in transplanted irrigated and rain fed lowland rice (Datta et al., 1996; Moody, 1991 and Rao et al; 2007). The weedy control had significantly highest weed density and dry weed weight over all the treatments. 
Table 1.a. Weed density at 30 DAS as influenced by integrated weed management practices in dry direct seeded rice at RARSs, Parwanipur, Bara

\begin{tabular}{|c|c|c|c|c|c|c|c|}
\hline \multirow[t]{3}{*}{ SNo } & \multirow[t]{3}{*}{ Treatments } & \multicolumn{6}{|c|}{ Weed Density $\left(\mathrm{no} / \mathrm{m}^{2}\right) 30$ DAS } \\
\hline & & \multicolumn{2}{|c|}{ Broad Leaves } & \multicolumn{2}{|c|}{ Sedges } & \multicolumn{2}{|c|}{ Grasses } \\
\hline & & 2010 & 2011 & 2010 & 2011 & 2010 & 2011 \\
\hline 1 & Weedy & $\begin{array}{l}112.40 \mathrm{~A} \\
(10.22 \mathrm{~A})\end{array}$ & $\begin{array}{l}15.10 \mathrm{~B} \\
(3.94 \mathrm{~A})\end{array}$ & $\begin{array}{l}40.60 \mathrm{~B} \\
(6.41 \mathrm{~B})\end{array}$ & $\begin{array}{c}12.60 \mathrm{AB} \\
(3.61 \mathrm{~A})\end{array}$ & $\begin{array}{l}104.40 \mathrm{~A} \\
(10.15 \mathrm{~A})\end{array}$ & $\begin{array}{l}12.76 \mathrm{~B} \\
(3.64 \mathrm{~A})\end{array}$ \\
\hline 2 & Weed free & $0.00 \mathrm{C}(0.71 \mathrm{D})$ & $\begin{array}{l}0.00 \mathrm{~B} \\
(0.71 \mathrm{~F})\end{array}$ & $\begin{array}{c}0.00 \mathrm{E} \\
(0.71 \mathrm{H})\end{array}$ & $\begin{array}{l}0.00 \mathrm{AB} \\
(0.71 \mathrm{~F})\end{array}$ & $\begin{array}{c}0.00 \mathrm{C} \\
(0.71 \mathrm{~F})\end{array}$ & $\begin{array}{l}0.00 \mathrm{~B} \\
(0.71 \mathrm{~F})\end{array}$ \\
\hline 3 & $\begin{array}{l}\text { Pendimethalin } \\
\text { fb Bispyribac }\end{array}$ & $\begin{array}{l}68.60 \mathrm{~B} \\
(7.98 \mathrm{~B})\end{array}$ & $\begin{array}{l}11.45 \mathrm{C} \\
(3.45 \mathrm{~B})\end{array}$ & $\begin{array}{l}146.90 \mathrm{~A} \\
(12.14 \mathrm{~A})\end{array}$ & $\begin{array}{l}9.08 \mathrm{C} \\
(3.09 \mathrm{~B})\end{array}$ & $\begin{array}{l}62.00 \mathrm{~B} \\
(7.90 \mathrm{~B})\end{array}$ & $\begin{array}{c}11.68 \mathrm{D} \\
(3.48 \mathrm{AB})\end{array}$ \\
\hline 4 & $\begin{array}{l}\text { Pendimethalin } \\
\text { fb two hand } \\
\text { weeding }\end{array}$ & $\begin{array}{c}7.40 \mathrm{C} \\
(2.51 \mathrm{CD})\end{array}$ & $\begin{array}{c}0.50 \mathrm{C} \\
(0.99 \mathrm{E})\end{array}$ & $\begin{array}{c}1.30 \mathrm{DE} \\
(1.33 \mathrm{FG})\end{array}$ & $\begin{array}{c}0.43 \mathrm{~B} \\
(0.96 \mathrm{~F})\end{array}$ & $\begin{array}{l}4.80 \mathrm{C} \\
(2.30 \mathrm{E})\end{array}$ & $\begin{array}{c}3.38 \mathrm{C} \\
(1.97 \mathrm{D})\end{array}$ \\
\hline 5 & $\begin{array}{l}\text { Stale seedbed } \\
\text { fb Bispyribac }\end{array}$ & $8.90 \mathrm{C}(2.80 \mathrm{C})$ & $\begin{array}{c}1.08 \mathrm{~B} \\
(1.24 \mathrm{D})\end{array}$ & $\begin{array}{l}4.50 \mathrm{DE} \\
(2.23 \mathrm{E})\end{array}$ & $\begin{array}{l}3.83 \mathrm{AB} \\
(2.07 \mathrm{D})\end{array}$ & $\begin{array}{c}10.80 \mathrm{C} \\
(3.35 \mathrm{D})\end{array}$ & $\begin{array}{c}6.88 \mathrm{~B} \\
(2.71 \mathrm{C})\end{array}$ \\
\hline 6 & $\begin{array}{l}\text { Stale seedbed } \\
\mathrm{fb} \\
\text { Pendimethalin } \\
\text { fb Bispyribac }\end{array}$ & $\begin{array}{c}8.20 \mathrm{C} \\
(2.49 \mathrm{CD})\end{array}$ & $\begin{array}{c}0.95 \mathrm{~B} \\
(1.20 \mathrm{DE})\end{array}$ & $\begin{array}{l}1.50 \mathrm{DE} \\
(1.41 \mathrm{~F})\end{array}$ & $\begin{array}{l}3.40 \mathrm{AB} \\
(1.97 \mathrm{D})\end{array}$ & $\begin{array}{c}9.70 \mathrm{C} \\
(3.19 \mathrm{DE})\end{array}$ & $\begin{array}{l}4.43 \mathrm{~B} \\
(2.22 \mathrm{D})\end{array}$ \\
\hline 7 & $\begin{array}{l}\text { Mulch } 4 \mathrm{t} / \mathrm{ha} \\
\mathrm{fb} \text { Bispyribac } \\
\text { fb 0ne hand } \\
\text { weeding }\end{array}$ & $\begin{array}{l}13.10 \mathrm{C} \\
(3.34 \mathrm{C})\end{array}$ & $\begin{array}{c}8.03 \mathrm{C} \\
(2.92 \mathrm{C})\end{array}$ & $\begin{array}{l}6.80 \mathrm{D} \\
(2.68 \mathrm{D})\end{array}$ & $\begin{array}{c}5.48 \mathrm{C} \\
(2.44 \mathrm{C})\end{array}$ & $\begin{array}{l}18.80 \mathrm{C} \\
(4.39 \mathrm{C})\end{array}$ & $\begin{array}{c}10.28 \mathrm{DE} \\
(3.27 \mathrm{~B})\end{array}$ \\
\hline 8 & $\begin{array}{l}\text { Stale seedbed } \\
\mathrm{fb} \text { mulch } \\
4 \mathrm{t} / \mathrm{ha} \mathrm{fb} \\
\text { Bispyribac }\end{array}$ & $\begin{array}{l}56.90 \mathrm{~B} \\
(7.13 \mathrm{~B})\end{array}$ & $\begin{array}{c}0.78 \mathrm{C} \\
(1.13 \mathrm{DE})\end{array}$ & $\begin{array}{l}13.60 \mathrm{C} \\
(3.75 \mathrm{C})\end{array}$ & $\begin{array}{l}1.64 \mathrm{AB} \\
(1.45 \mathrm{E})\end{array}$ & $\begin{array}{l}53.10 \mathrm{~B} \\
(7.29 \mathrm{~B})\end{array}$ & $\begin{array}{l}3.65 \mathrm{~B} \\
(2.04 \mathrm{D})\end{array}$ \\
\hline 9 & $\begin{array}{l}\text { Pendimethalin } \\
\text { and Sesbania } \\
\text { co-culture fb } \\
\text { 2,4-D Na salt } \\
\text { fb one hand } \\
\text { weeding }\end{array}$ & $\begin{array}{l}63.60 \mathrm{~B} \\
(7.41 \mathrm{~B})\end{array}$ & $\begin{array}{l}11.05 \mathrm{~A} \\
(3.39 \mathrm{~B})\end{array}$ & $\begin{array}{l}15.30 \mathrm{C} \\
(3.96 \mathrm{C})\end{array}$ & $\begin{array}{l}8.10 \mathrm{~A} \\
(2.92 \mathrm{~B})\end{array}$ & $\begin{array}{l}65.10 \mathrm{~B} \\
(8.08 \mathrm{~B})\end{array}$ & $\begin{array}{c}11.78 \mathrm{~A} \\
(3.50 \mathrm{AB})\end{array}$ \\
\hline 10 & $\begin{array}{l}\text { Pendimethalin } \\
\mathrm{fb} 2,4-\mathrm{D} \mathrm{fb} \\
\text { one hand } \\
\text { weeding }\end{array}$ & $\begin{array}{c}5.74 \mathrm{C} \\
(2.32 \mathrm{CD})\end{array}$ & $\begin{array}{c}0.45 \mathrm{C} \\
0.97 \mathrm{E})\end{array}$ & $\begin{array}{c}0.50 \mathrm{E} \\
(1.00 \mathrm{GH})\end{array}$ & $\begin{array}{c}0.30 \mathrm{C} \\
(0.88 \mathrm{~F})\end{array}$ & $\begin{array}{c}4.60 \mathrm{C} \\
(2.26 \mathrm{E})\end{array}$ & $\begin{array}{c}1.88 \mathrm{E} \\
(1.53 \mathrm{E})\end{array}$ \\
\hline & Mean & $34.48(4.69)$ & $\begin{array}{c}4.94 \\
(1.99)\end{array}$ & $\begin{array}{l}23.10 \\
(12.14)\end{array}$ & $\begin{array}{c}4.48 \\
(2.01)\end{array}$ & $\begin{array}{l}33.33 \\
(4.96)\end{array}$ & $\begin{array}{c}6.67 \\
(2.50)\end{array}$ \\
\hline & $\mathrm{CV} \%$ & $64.61(26.47)$ & $\begin{array}{l}18.22 \\
(7.81)\end{array}$ & $\begin{array}{l}15.61 \\
(3.56)\end{array}$ & $\begin{array}{l}22.69 \\
(9.85)\end{array}$ & $\begin{array}{c}36.29 \\
(12.78)\end{array}$ & $\begin{array}{l}17.27 \\
(7.58)\end{array}$ \\
\hline & F-test & ** & $* *$ & $* *$ & $* *$ & $* *$ & $* *$ \\
\hline & $\mathrm{SD}$ at 0.05 & 32.33 & 1.306 & 5.232 & 1.476 & 17.55 & 1.67 \\
\hline
\end{tabular}

Note. $\mathrm{fb}=$ followed by, $\mathrm{hw}=$ hand weeding $\&$ figure in parentheses indicate square root transformed $(\sqrt{ } \mathrm{x}+0.5)$ original value 
Table 1. b. Weed density at 60 DAS as influenced by integrated weed management practices in dry direct seeded rice at Parwanipur, Bara

\begin{tabular}{|c|c|c|c|c|c|c|c|}
\hline \multirow{3}{*}{$\begin{array}{l}\text { S } \\
\text { No }\end{array}$} & \multirow[t]{3}{*}{ Treatments } & \multicolumn{6}{|c|}{ Weed Density $\left(\mathrm{no} / \mathrm{m}^{2}\right) 60$ DAS } \\
\hline & & \multicolumn{2}{|c|}{ Broad Leaves } & \multicolumn{2}{|c|}{ Sedges } & \multicolumn{2}{|c|}{ Grasses } \\
\hline & & 2010 & 2011 & 2010 & 2011 & 2010 & 2011 \\
\hline 1 & Weedy & $\begin{array}{l}66.70 \mathrm{~A} \\
(6.74 \mathrm{~A})\end{array}$ & $\begin{array}{c}12.18 \mathrm{ABC} \\
(3.56 \mathrm{~A})\end{array}$ & $\begin{array}{l}16.50 \mathrm{~A} \\
(4.11 \mathrm{~A})\end{array}$ & $\begin{array}{c}21.80 \mathrm{AB} \\
(4.72 \mathrm{~A})\end{array}$ & $\begin{array}{l}51.00 \mathrm{~A} \\
(7.17 \mathrm{~A})\end{array}$ & $\begin{array}{l}18.93 \mathrm{~B} \\
(4.41 \mathrm{~A})\end{array}$ \\
\hline 2 & Weed free & $\begin{array}{c}0.00 \mathrm{~B} \\
(0.71 \mathrm{C})\end{array}$ & $\begin{array}{l}0.00 \mathrm{BC} \\
(0.71 \mathrm{D})\end{array}$ & $\begin{array}{c}0.00 \mathrm{E} \\
(0.71 \mathrm{G})\end{array}$ & $\begin{array}{c}0.00 \mathrm{~B} \\
(0.71 \mathrm{G})\end{array}$ & $\begin{array}{c}0.00 \mathrm{~F} \\
(0.71 \mathrm{G})\end{array}$ & $\begin{array}{c}0.00 \mathrm{~B} \\
(0.71 \mathrm{~F})\end{array}$ \\
\hline 3 & $\begin{array}{l}\text { Pendimethalin } \mathrm{fb} \\
\text { Bispyribac }\end{array}$ & $\begin{array}{l}44.20 \mathrm{~A} \\
(6.64 \mathrm{~A})\end{array}$ & $\begin{array}{l}11.08 \mathrm{~A} \\
(3.39 \mathrm{~A})\end{array}$ & $\begin{array}{l}12.10 \mathrm{~B} \\
(3.53 \mathrm{~B})\end{array}$ & $\begin{array}{c}16.50 \mathrm{~A} \\
(4.12 \mathrm{BC})\end{array}$ & $\begin{array}{l}32.80 \mathrm{C} \\
(5.76 \mathrm{~B})\end{array}$ & $\begin{array}{l}16.50 \mathrm{~A} \\
(4.12 \mathrm{~A})\end{array}$ \\
\hline 4 & $\begin{array}{l}\text { Pendimet } \\
\text { two hand }\end{array}$ & $\begin{array}{c}2.80 \mathrm{~B} \\
(1.70 \mathrm{BC})\end{array}$ & $\begin{array}{r}5.05 \mathrm{~A} \\
(2.35\end{array}$ & $\begin{array}{c}0.80 \mathrm{DE} \\
(1.11 \mathrm{EFG})\end{array}$ & $\begin{array}{l}9.90 \mathrm{AB} \\
(3.21 \mathrm{E})\end{array}$ & $\begin{array}{l}1.11 \mathrm{~F} \\
(1.27 \mathrm{~F})\end{array}$ & $\begin{array}{l}3.55 \mathrm{~B} \\
(2.01 \mathrm{E})\end{array}$ \\
\hline 5 & $\begin{array}{l}\text { Stale seedbed } \mathrm{fb} \\
\text { Bispyribac }\end{array}$ & $\begin{array}{c}3.80 \mathrm{~B} \\
(2.03 \mathrm{BC})\end{array}$ & $\begin{array}{l}10.13 \mathrm{~A} \\
(3.25 \mathrm{~A}\end{array}$ & $\begin{array}{l}1.80 \mathrm{DE} \\
(1.51 \mathrm{DE})\end{array}$ & $\begin{array}{r}15.3 \\
(3.97\end{array}$ & $\begin{array}{l}4.60 \mathrm{~F} \\
(2.22 \mathrm{E})\end{array}$ & $\begin{array}{c}9.93 \mathrm{~B} \\
(3.22 \mathrm{C})\end{array}$ \\
\hline 6 & $\begin{array}{l}\text { Stale seedbed } \mathrm{fb} \\
\text { Pendimethalin } \mathrm{fb} \\
\text { Bispyribac }\end{array}$ & $\begin{array}{c}3.10 \mathrm{~B} \\
(1.81 \mathrm{BC})\end{array}$ & $\begin{array}{c}8.60 \mathrm{C} \\
(3.01 \mathrm{~B})\end{array}$ & $\begin{array}{l}1.10 \mathrm{DE} \\
(1.26 \mathrm{EF})\end{array}$ & $\begin{array}{c}14.20 \mathrm{~B} \\
(3.83 \mathrm{CD})\end{array}$ & $\begin{array}{l}1.90 \mathrm{~F} \\
(1.53 \mathrm{~F})\end{array}$ & $\begin{array}{c}9.23 \mathrm{~B} \\
(3.11 \mathrm{C})\end{array}$ \\
\hline 7 & $\begin{array}{l}\text { Mulch } 4 \mathrm{t} / \mathrm{ha} \mathrm{fb} \\
\text { Bispyribac fb 0ne } \\
\text { hand weeding }\end{array}$ & $\begin{array}{c}8.00 \mathrm{~B} \\
(2.85 \mathrm{~B})\end{array}$ & $\begin{array}{c}10.40 \mathrm{E} \\
(3.30 \mathrm{AB})\end{array}$ & $\begin{array}{l}3.10 \mathrm{D} \\
(1.87 \mathrm{D})\end{array}$ & $\begin{array}{c}16.38 \mathrm{D} \\
(4.10 \mathrm{BC})\end{array}$ & $\begin{array}{c}9.30 \mathrm{E} \\
(3.12 \mathrm{D})\end{array}$ & $\begin{array}{l}12.40 \mathrm{D} \\
(3.59 \mathrm{~B})\end{array}$ \\
\hline 8 & $\begin{array}{l}\text { Stale seedbed fb } \\
\text { mulch4t/ha fb } \\
\text { Bispyribac }\end{array}$ & $\begin{array}{l}33.10 \mathrm{~A} \\
(5.57 \mathrm{~A})\end{array}$ & $\begin{array}{l}8.50 \mathrm{AB} \\
(2.99 \mathrm{~B})\end{array}$ & $\begin{array}{c}8.30 \mathrm{C} \\
(2.93 \mathrm{C})\end{array}$ & $\begin{array}{l}12.18 \mathrm{AB} \\
(3.55 \mathrm{DE})\end{array}$ & $\begin{array}{l}24.60 \mathrm{D} \\
(5.00 \mathrm{C})\end{array}$ & $\begin{array}{c}6.23 \mathrm{~B} \\
(2.58 \mathrm{D})\end{array}$ \\
\hline 9 & $\begin{array}{l}\text { Pendimethalin and } \\
\text { Sesbania co-culture } \\
\mathrm{fb} 2,4-\mathrm{D} \text { Na salt } \mathrm{fb} \\
\text { one hand weeding }\end{array}$ & $\begin{array}{l}45.90 \mathrm{~A} \\
(6.50 \mathrm{~A})\end{array}$ & $\begin{array}{l}11.78 \mathrm{D} \\
(3.50 \mathrm{~A})\end{array}$ & $\begin{array}{l}12.80 \mathrm{~B} \\
(3.63 \mathrm{~B})\end{array}$ & $\begin{array}{l}17.85 \mathrm{C} \\
(4.28 \mathrm{~B})\end{array}$ & $\begin{array}{l}37.30 \mathrm{~B} \\
(6.14 \mathrm{~B})\end{array}$ & $\begin{array}{l}16.85 \mathrm{C} \\
(4.16 \mathrm{~A})\end{array}$ \\
\hline 10 & $\begin{array}{l}\text { Pendimethalin } \mathrm{fb} \\
2,4-\mathrm{D} \text { fb one hand } \\
\text { weeding }\end{array}$ & $\begin{array}{c}1.80 \mathrm{~B} \\
(1.32 \mathrm{C})\end{array}$ & $\begin{array}{c}3.73 \mathrm{E} \\
(2.05 \mathrm{C})\end{array}$ & $\begin{array}{c}0.30 \mathrm{DE} \\
(0.86 \mathrm{FG})\end{array}$ & $\begin{array}{c}6.65 \mathrm{D} \\
(2.67 \mathrm{~F})\end{array}$ & $\begin{array}{c}0.90 \mathrm{~F} \\
(1.17 \mathrm{FG})\end{array}$ & $\begin{array}{l}3.13 \mathrm{D} \\
(1.89 \mathrm{E})\end{array}$ \\
\hline & Mean & $\begin{array}{l}18.94 \\
(3.58)\end{array}$ & $\begin{array}{c}8.14 \\
(2.81)\end{array}$ & $\begin{array}{l}5.68 \\
(2.15)\end{array}$ & $\begin{array}{l}13.08 \\
(3.52)\end{array}$ & $\begin{array}{l}16.35 \\
(3.41)\end{array}$ & $\begin{array}{c}9.67 \\
(2.98)\end{array}$ \\
\hline & $\mathrm{CV} \%$ & $\begin{array}{c}60.17 \\
(26.22)\end{array}$ & $\begin{array}{l}16.37 \\
(7.63)\end{array}$ & $\begin{array}{c}32.79 \\
(14.51)\end{array}$ & $\begin{array}{l}14.96 \\
(7.42)\end{array}$ & $\begin{array}{l}18.50 \\
(9.65)\end{array}$ & $\begin{array}{l}14.41 \\
(6.77)\end{array}$ \\
\hline & & $* *$ & $* *$ & & $* *$ & $* *$ & $* *$ \\
\hline & LSD at 0.05 & 16.53 & 1.934 & 2.702 & 2.837 & 4.388 & 2.023 \\
\hline
\end{tabular}

Note: $\mathrm{fb}=$ followed by, hw=hand weeding $\&$ figure in parentheses indicate square root transformed $(\sqrt{ } \mathrm{x}+0.5)$ original value 
Table 2.a. Dry weed weight at 30 DAS as influenced by integrated weed management practices in dry direct seeded rice at Pparwanipur, Bara

\begin{tabular}{|c|c|c|c|c|c|c|c|}
\hline \multirow[t]{3}{*}{ SNo } & \multirow[t]{3}{*}{ Treatments } & \multicolumn{6}{|c|}{ Dry weed weight $\left(\mathrm{g} / \mathrm{m}^{2}\right) 30$ DAS } \\
\hline & & \multicolumn{2}{|c|}{ Broad Leaves } & \multicolumn{2}{|c|}{ Sedges } & \multicolumn{2}{|c|}{ Grasses } \\
\hline & & 2010 & 2011 & 2010 & 2011 & 2010 & 2011 \\
\hline 1 & Weedy & $\begin{array}{l}19.72 \mathrm{~A} \\
(4.34 \mathrm{a})\end{array}$ & $\begin{array}{c}2.65 \mathrm{~B} \\
(1.77 \mathrm{~A})\end{array}$ & $\begin{array}{l}7.13 \mathrm{~A} \\
(2.96 \mathrm{~B})\end{array}$ & $\begin{array}{l}2.21 \mathrm{AB} \\
(1.65 \mathrm{~A})\end{array}$ & $\begin{array}{l}18.32 \mathrm{~A} \\
(4.30 \mathrm{~A})\end{array}$ & $\begin{array}{c}2.24 \mathrm{~B} \\
(1.66 \mathrm{~A})\end{array}$ \\
\hline 2 & Weed free & $\begin{array}{c}0.00 \mathrm{C} \\
(0.71 \mathrm{C})\end{array}$ & $\begin{array}{c}0.00 \mathrm{~B} \\
(0.71 \mathrm{E})\end{array}$ & $\begin{array}{c}0.0 \mathrm{~B} \\
(0.71 \mathrm{~F})\end{array}$ & $\begin{array}{l}0.00 \mathrm{AB} \\
(0.71 \mathrm{~F})\end{array}$ & $\begin{array}{c}0.00 \mathrm{C} \\
(0.71 \mathrm{E})\end{array}$ & $\begin{array}{c}0.00 \mathrm{~B} \\
(0.71 \mathrm{~F})\end{array}$ \\
\hline 3 & $\begin{array}{l}\text { Pendimethalin } \mathrm{fb} \\
\text { Bispyribac }\end{array}$ & $\begin{array}{l}10.06 \mathrm{~B} \\
(2.86 \mathrm{~B})\end{array}$ & $\begin{array}{l}1.68 \mathrm{C} \\
(1.48 \mathrm{~B})\end{array}$ & $\begin{array}{l}21.55 \mathrm{C} \\
(4.70 \mathrm{~A})\end{array}$ & $\begin{array}{l}1.33 \mathrm{C} \\
(1.35 \mathrm{~B})\end{array}$ & $\begin{array}{l}9.09 \mathrm{~B} \\
(3.09 \mathrm{~B})\end{array}$ & $\begin{array}{l}1.71 \mathrm{D} \\
(1.49 \mathrm{~B})\end{array}$ \\
\hline 4 & $\begin{array}{l}\text { Pendimethalin fb two } \\
\text { hw }\end{array}$ & $\begin{array}{c}0.53 \mathrm{C} \\
(1.00 \mathrm{C})\end{array}$ & $\begin{array}{c}0.07 \mathrm{~B} \\
(0.75 \mathrm{DE})\end{array}$ & $\begin{array}{c}0.27 \mathrm{E} \\
(0.88 \mathrm{E})\end{array}$ & $\begin{array}{l}0.23 \mathrm{AB} \\
(0.85 \mathrm{E})\end{array}$ & $\begin{array}{c}0.64 \mathrm{C} \\
(1.07 \mathrm{DEF})\end{array}$ & $\begin{array}{l}0.41 \mathrm{~B} \\
(0.95 \mathrm{D})\end{array}$ \\
\hline 5 & $\begin{array}{l}\text { Stale seedbed } \mathrm{fb} \\
\text { Bispyribac }\end{array}$ & $\begin{array}{c}1.14 \mathrm{C} \\
(1.19 \mathrm{C})\end{array}$ & $\begin{array}{c}0.13 \mathrm{~B} \\
(0.79 \mathrm{D})\end{array}$ & $\begin{array}{r}0.21 \mathrm{DE} \\
(0.84 \mathrm{EF})\end{array}$ & $\begin{array}{l}0.47 \mathrm{AB} \\
(0.99 \mathrm{D})\end{array}$ & $\begin{array}{c}1.35 \mathrm{C} \\
(1.36 \mathrm{D})\end{array}$ & $\begin{array}{c}0.61 \mathrm{~B} \\
(1.06 \mathrm{C})\end{array}$ \\
\hline 6 & $\begin{array}{l}\text { Stale seedbed } \mathrm{fb} \\
\text { Pendimethalin } \mathrm{fb} \\
\text { Bispyribac }\end{array}$ & $\begin{array}{c}1.01 \mathrm{C} \\
(1.48 \mathrm{C})\end{array}$ & $\begin{array}{c}0.08 \mathrm{C} \\
(0.76 \mathrm{DE})\end{array}$ & $\begin{array}{c}0.09 \mathrm{D} \\
(0.77 \mathrm{EF})\end{array}$ & $\begin{array}{c}0.06 \mathrm{C} \\
(0.74 \mathrm{~F})\end{array}$ & $\begin{array}{c}0.81 \mathrm{C} \\
(1.15 \mathrm{DE})\end{array}$ & $\begin{array}{c}0.33 \mathrm{E} \\
(0.91 \mathrm{DE})\end{array}$ \\
\hline 7 & $\begin{array}{l}\text { Mulch } 4 \mathrm{t} / \mathrm{ha} \mathrm{fb} \\
\text { Bispyribac fb one hw }\end{array}$ & $\begin{array}{c}1.92 \mathrm{C} \\
(1.48 \mathrm{C})\end{array}$ & $\begin{array}{c}1.18 \mathrm{C} \\
(1.30 \mathrm{C})\end{array}$ & $\begin{array}{l}1.00 \mathrm{DE} \\
(1.22 \mathrm{D})\end{array}$ & $\begin{array}{c}0.81 \mathrm{C} \\
(1.14 \mathrm{C})\end{array}$ & $\begin{array}{c}2.76 \mathrm{C} \\
(1.80 \mathrm{C})\end{array}$ & $\begin{array}{l}1.51 \mathrm{E} \\
(1.41 \mathrm{~B})\end{array}$ \\
\hline 8 & $\begin{array}{l}\text { Stale seedbed fb } \\
\text { mulch } 4 \mathrm{t} / \mathrm{ha} \mathrm{fb} \\
\text { Bispyribac }\end{array}$ & $\begin{array}{l}9.98 \mathrm{~B} \\
(1.20 \mathrm{~B})\end{array}$ & $\begin{array}{c}0.14 \mathrm{C} \\
(0.80 \mathrm{D})\end{array}$ & $\begin{array}{c}2.39 \mathrm{E} \\
(1.70 \mathrm{C})\end{array}$ & $\begin{array}{c}0.29 \mathrm{C} \\
(0.89 \mathrm{E})\end{array}$ & $\begin{array}{l}9.32 \mathrm{~B} \\
(3.12 \mathrm{~B})\end{array}$ & $\begin{array}{l}0.64 \mathrm{CD} \\
(1.07 \mathrm{C})\end{array}$ \\
\hline 9 & $\begin{array}{l}\text { Pendimethalin and } \\
\text { Sesbania co-culture fb } \\
\text { 2,4-D Na salt fb one } \\
\text { hw }\end{array}$ & $\begin{array}{l}8.83 \mathrm{~B} \\
(0.95 \mathrm{~B})\end{array}$ & $\begin{array}{c}1.54 \mathrm{~A} \\
(1.42 \mathrm{~B})\end{array}$ & $\begin{array}{c}2.13 \mathrm{E} \\
(1.62 \mathrm{C})\end{array}$ & $\begin{array}{c}1.13 \mathrm{~A} \\
(1.27 \mathrm{~B})\end{array}$ & $\begin{array}{c}9.04 \mathrm{~B} \\
(3.08 \mathrm{~B})\end{array}$ & $\begin{array}{c}1.64 \mathrm{~A} \\
(1.46 \mathrm{~B})\end{array}$ \\
\hline 10 & $\begin{array}{l}\text { Pendimethalin fb 2,4- } \\
\text { D fb one hw }\end{array}$ & $\begin{array}{c}0.44 \mathrm{C} \\
(3.07 \mathrm{C})\end{array}$ & $\begin{array}{c}0.03 \mathrm{C} \\
(0.73 \mathrm{DE})\end{array}$ & $\begin{array}{c}0.08 \mathrm{C} \\
(0.76 \mathrm{EF})\end{array}$ & $\begin{array}{c}0.03 \mathrm{~B} \\
(0.73 \mathrm{~F})\end{array}$ & $\begin{array}{c}0.29 \mathrm{C} \\
(0.89 \mathrm{EF})\end{array}$ & $\begin{array}{c}0.20 \mathrm{C} \\
(0.84 \mathrm{E})\end{array}$ \\
\hline & Mean & $\begin{array}{c}5.36 \\
(1.99)\end{array}$ & $\begin{array}{c}0.75 \\
(1.05)\end{array}$ & $\begin{array}{c}3.48 \\
(1.59)\end{array}$ & $\begin{array}{c}0.65 \\
(1.03)\end{array}$ & $\begin{array}{c}5.16 \\
(2.06)\end{array}$ & $\begin{array}{c}0.93 \\
(1.15)\end{array}$ \\
\hline & $\mathrm{CV} \%$ & $\begin{array}{c}70.25 \\
(26.65)\end{array}$ & $\begin{array}{l}17.78 \\
(4.22)\end{array}$ & $\begin{array}{l}15.71 \\
(5.80)\end{array}$ & $\begin{array}{l}23.05 \\
(5.79)\end{array}$ & $\begin{array}{c}40.28 \\
(12.28)\end{array}$ & $\begin{array}{l}17.63 \\
(5.19)\end{array}$ \\
\hline & F-te & $* *$ & $* *$ & $* *$ & $* *$ & $* *$ & $* *$ \\
\hline & LSD at 0.05 & 5.465 & 0.1947 & 0.7827 & 0.22 & 30.16 & 0.2384 \\
\hline
\end{tabular}

Note. $\mathrm{fb}=$ followed by, $\mathrm{hw}=$ hand weeding $\&$ figure in parentheses indicate square root transformed $(\sqrt{ } \mathrm{x}+0.5)$ original value 
Table 2.b. Dry weed weight at $60 \mathrm{DAD}$ as influenced by integrated weed management practices in dry direct seeded rice at Parwanipur, Bara

\begin{tabular}{|c|c|c|c|c|c|c|c|}
\hline \multirow[t]{3}{*}{ SN } & \multirow[t]{3}{*}{ Treatments } & \multicolumn{6}{|c|}{ Dry weed weight $\left(\mathrm{g} / \mathrm{m}^{2}\right) 60$ DAS } \\
\hline & & \multicolumn{2}{|c|}{ Broad Leaves } & \multicolumn{2}{|c|}{ Sedges } & \multicolumn{2}{|c|}{ Grasses } \\
\hline & & 2010 & 2011 & 2010 & 2011 & 2010 & 2011 \\
\hline \multirow[t]{2}{*}{1} & Weedy & $42.46 \mathrm{~A}$ & $11.28 \mathrm{~B}$ & $15.43 \mathrm{~A}$ & $20.22 \mathrm{~B}$ & $47.53 \mathrm{~A}$ & $17.58 \mathrm{~A}$ \\
\hline & & $(6.45 \mathrm{~A})$ & $(3.43 \mathrm{~A})$ & $(3.9$ & $(4.5$ & $(6.92 \mathrm{~A})$ & $(4.25 \mathrm{~A})$ \\
\hline \multirow[t]{2}{*}{2} & Weed free & $0.00 \mathrm{~B}$ & $0.00 \mathrm{~B}$ & $0.00 \mathrm{D}$ & $0.00 \mathrm{~B}$ & $0.00 \mathrm{E}$ & $0.00 \mathrm{~A}$ \\
\hline & & $(0.71 \mathrm{C})$ & $(0.71 \mathrm{~F})$ & $(0.71 \mathrm{G})$ & $(0.71 \mathrm{G})$ & $(0.71 \mathrm{G})$ & $(0.71 \mathrm{~F})$ \\
\hline \multirow[t]{2}{*}{3} & Pendimethalin $\mathrm{fb}$ & $46.13 \mathrm{~A}$ & $11.56 \mathrm{~B}$ & $12.63 \mathrm{AB}$ & $17.22 \mathrm{AB}$ & $34.23 \mathrm{~B}$ & $17.22 \mathrm{~A}$ \\
\hline & Bispy & $(6.78 \mathrm{~A})$ & $(3.46 \mathrm{~A})$ & $(3.61$ & $(4.20 \mathrm{AB})$ & 8B) & $(4.20 \mathrm{~A})$ \\
\hline \multirow[t]{2}{*}{4} & Pendimet & $3.02 \mathrm{~B}$ & $5.44 \mathrm{AB}$ & 0.8 & 10.67 & $1.20 \mathrm{E}$ & \\
\hline & two hw & $(1.75 \mathrm{BC})$ & $(2.43 \mathrm{D})$ & $(1.14 \mathrm{EFG})$ & $(3.33$ & $(1.30 \mathrm{~F})$ & $(2.08 \mathrm{E})$ \\
\hline \multirow[t]{2}{*}{5} & Stale seedbed $\mathrm{fb}$ & $4.10 \mathrm{~B}$ & $10.91 \mathrm{~B}$ & $1.94 \mathrm{D}$ & $16.49 \mathrm{~B}$ & $4.96 \mathrm{DE}$ & $10.70 \mathrm{~A}$ \\
\hline & Bispy & $(2.09 \mathrm{BC})$ & $(3.37 \mathrm{AB})$ & $(1.56 \mathrm{DE})$ & $(4.11 \mathrm{~B}$ & $(2.30 \mathrm{E})$ & $(3.34 \mathrm{C})$ \\
\hline \multirow[t]{2}{*}{6} & Stale seedbed $\mathrm{fb}$ & $3.24 \mathrm{~B}$ & 8.98B & $1.15 \mathrm{D}$ & 14.82B & $1.98 \mathrm{E}$ & $9.63 \mathrm{~A}$ \\
\hline & $\begin{array}{l}\text { Pendimethalin fb } \\
\text { Bispyribac }\end{array}$ & $(1.84 \mathrm{BC})$ & $(3.07 \mathrm{BC})$ & (1.28DEF) & (3.91BCD) & $(1.55 \mathrm{~F})$ & $(3.18 \mathrm{C})$ \\
\hline \multirow[t]{2}{*}{7} & Mulch 4 t/ha fb & $6.64 \mathrm{~B}$ & $8.63 \mathrm{D}$ & & 13.5 & $7.72 \mathrm{D}$ & \\
\hline & $\begin{array}{l}\text { Bispyribac fb one } \\
\text { hw }\end{array}$ & $(2.61 \mathrm{~B})$ & & & & & \\
\hline \multirow[t]{2}{*}{8} & Stale seedbed $\mathrm{fb}$ & 33.9 & $8.71 \mathrm{~A}$ & $8.50 \mathrm{C}$ & 12.47 & $25.20 \mathrm{C}$ & 6.38 \\
\hline & & $(5.63 \mathrm{~A})$ & $(3.03 C)$ & & (3.59DE) & $(5.06 \mathrm{C})$ & $(2.61 \mathrm{E})$ \\
\hline \multirow[t]{3}{*}{9} & Pendimethalin and & $38.08 \mathrm{~A}$ & $9.77 \mathrm{C}$ & 10.62 & 14.81 & $30.94 \mathrm{~B}$ & $13.98 \mathrm{~B}$ \\
\hline & Sesbania co- & $(5.93 \mathrm{~A})$ & $(3.20 \mathrm{ABC})$ & & (3.91BCD) & $(2.60 \mathrm{~B})$ & \\
\hline & $\mathrm{Na}$ sa & & & & & & \\
\hline \multirow[t]{8}{*}{10} & Pendimethalin $\mathrm{fb}$ & $1.62 \mathrm{~B}$ & $3.34 \mathrm{D}$ & $0.27 \mathrm{D}$ & $5.96 \mathrm{D}$ & $0.81 \mathrm{E}$ & $2.80 \mathrm{C}$ \\
\hline & 2,4-D fb one hw & $(1.28 \mathrm{BC})$ & $(1.96 \mathrm{E})$ & (0.85FG) & $(2.54 \mathrm{~F})$ & (1.14FG) & $(1.81 \mathrm{E})$ \\
\hline & Mean & 17.92 & 7.86 & 5.40 & 12.62 & 15.46 & 9.24 \\
\hline & & $(3.51)$ & & & & (3.33) & $(2.92)$ \\
\hline & $\mathrm{CV} \%$ & 56.05 & 17.31 & 35.95 & 15.72 & 22.79 & 15.98 \\
\hline & & $(25.20)$ & & & & (10.59) & \\
\hline & & $* *$ & $* *$ & $* *$ & $* *$ & $* *$ & $* *$ \\
\hline & LSD at 0.05 & 14.57 & 1.974 & 2.814 & 2.88 & 5.11 & 2.142 \\
\hline
\end{tabular}

Note. $\mathrm{fb}=$ followed by, $\mathrm{hw}=$ hand weeding $\&$ figure in parentheses indicate square root transformed $(\sqrt{ } \mathrm{x}+0.5)$ original value

\section{Effect on crop yield and yield attributes.}

The weed free treatment produced maximum yield of direct seeded rice in both years. This might be attributed to better growth of plants on account of reduced weed competition at critical crop growth stages resulting in increased availability of nutrients, water and light. All the weed control treatments significantly increased the number of panicles $/ \mathrm{m}^{2}$, panicle weight, filled grains/panicle and thousand grain weight and ultimately the yield over weedy check except unfilled grain and 1000 grain weight in 2011, which were non significant. Among the herbicidal treatments, the use of 
Pendimethalin $\mathrm{fb}$ 2,4-D $\mathrm{fb}$ one hand weeding produced maximum number of panicles $/ \mathrm{m}^{2}$ (230) panicle weight, filled grains/panicle and 1000 grain weight and yield during both years (table-3) which were comparable to that of weed free treatment, Samar Singh et al; (2005) reported similar results with the use of Pendimethalin in dry direct seeded rice.

Table 3. Effect of integrated weed management practices on yield attributes of dry direct seeded rice

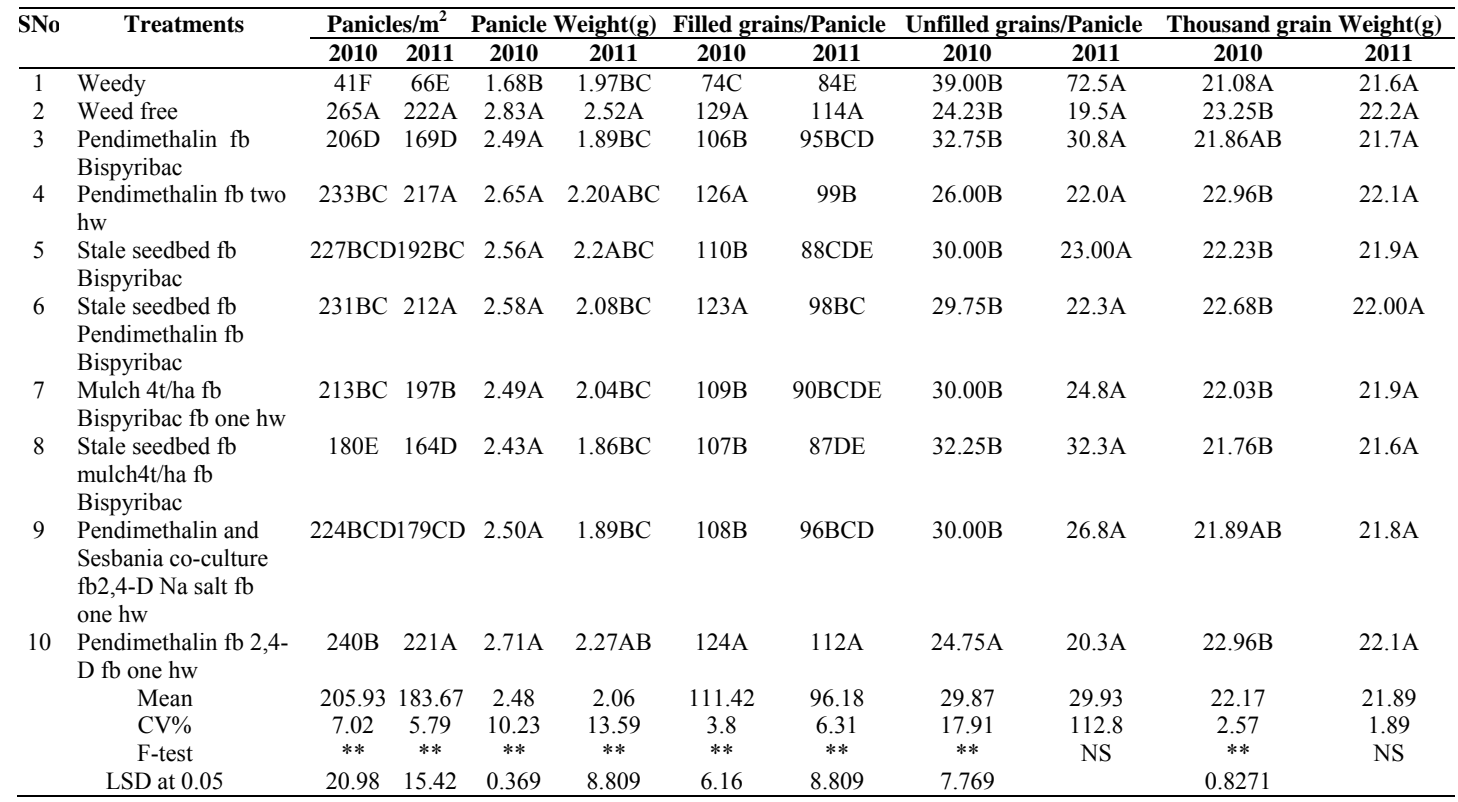

The highest grain yield (5305 in 2010\& $6369 \mathrm{~kg} / \mathrm{ha}$ in 2011) was recorded at weed free treatment in both years whereas, use of Pendimethalin $\mathrm{fb}$ 2,4-D $\mathrm{fb}$ one hand weeding which also showed statistically at par with weed free. This was perhaps due to high weed control efficiency of the treatment (Pendimethalin $\mathrm{fb} 2,4-\mathrm{D} \mathrm{fb}$ one hand weeding). It was closely followed by the use of Pendimethalin $\mathrm{fb}$ two hand weeding and stale seed bed $\mathrm{fb}$ Pendimethalin $\mathrm{fb}$ Bispyribac. In each case, the involvement of Pendimethalin followed by manual weeding or other herbicide indicates that Pendimethalin seems to be an effective pre-emergence herbicide for weed control in direct seeded rice. The efficacy of Pendimethalin alone is high reported by several authors (Moody,1991; and Valverde et al; 2001) or in combination with hand weeding was reported so effective in controlling weeds in dry direct seeded rice (Ramamoorthy et al; 1998 and Singh et al; 2005). Rainfall pattern of monsoon in the second year was well distributed during crop growth period resulted better crop performance. The experimental plots were heavily infested with rice Gundhi bug (Leptocorisa varicornis) which subsequently reduced yield in 2010. 


\section{Economics}

A perusal of data on economic analysis of integrated weed management practices in dry direct seeded rice suggested that cost of cultivation of weed free treatment was almost same in both years due to this low net return. However, net returns observed almost double in the year 2011 than 2010 (Table-4) due to more production. The highest yield resulted in weed free check followed by Pendimethalin followed by two hand weeding and Pendimethalin followed by 2,4-D followed by one hand weeding. However, the net return per unit investment resulted highest in Pendimethalin followed by 2,4-D followed by one hand weeding. The net return per unit investment exceed by stale seed bed followed by Bispyribac and stale seed bed followed by Pendimethalin followed by Bispyribac than weed free check. This revealed that amid increasing wage rate and labour scarcity integrated weed management through Pendimethalin 30 EC (stomp)@ $1 \mathrm{~kg}$ a. i./ha as preemergence herbicide application followed by 2, 4- D sodium salt $80 \mathrm{WP} @ 0.5 \mathrm{Kg}$ a. i. /ha followed by one hand weeding or stale seed bed followed by Pendimethalin 30 EC (stomp) @1 kg a. i./ha followed by Bispyribac (nominee gold) @ $25 \mathrm{~g}$ a. i./ha $10 \%$ @ $200 \mathrm{ml} / \mathrm{ha}$ at 20 days of seeding resulted best alternative for manual hand weeding practices giving higher net return per unit investment.

Table 4. Grain yield and net return as influenced by different weed management treatments at RARS, Parwanipur

\begin{tabular}{|c|c|c|c|c|c|c|c|}
\hline \multirow[t]{2}{*}{ SNo } & \multirow[t]{2}{*}{ Treatments } & \multicolumn{2}{|c|}{ Grain Yield (kg/ha) } & \multicolumn{2}{|c|}{$\begin{array}{l}\text { Benefit Cost } \\
\text { Ratio }\end{array}$} & \multicolumn{2}{|c|}{$\begin{array}{l}\text { Net Return/Rs } \\
\text { Investment }\end{array}$} \\
\hline & & 2010 & 2011 & 2010 & 2011 & 2010 & 2011 \\
\hline 1 & Weedy & $537 \mathrm{~F}$ & $2065 \mathrm{G}$ & 0.30 & 0.95 & -0.70 & -0.05 \\
\hline 2 & Weed free & $5305 \mathrm{~A}$ & $6369 \mathrm{~A}$ & 1.64 & 2.04 & 0.64 & 1.04 \\
\hline 3 & Pendimethalin fb Bispyribac & 3938DE & $5232 \mathrm{E}$ & 1.15 & 1.83 & 0.15 & 0.83 \\
\hline 4 & Pendimethalin fb two hw & 4997ABC & $6160 \mathrm{AB}$ & 1.64 & 2.16 & 0.64 & 1.16 \\
\hline 5 & Stale seedbed fb Bispyribac & $4525 \mathrm{BCD}$ & $5871 \mathrm{BCD}$ & 1.56 & 2.11 & 0.56 & 1.11 \\
\hline 6 & $\begin{array}{l}\text { Stale seedbed } \mathrm{fb} \\
\text { Pendimethalin fb Bispyribac }\end{array}$ & $4971 \mathrm{ABC}$ & $6134 \mathrm{ABC}$ & 1.64 & 2.12 & 0.64 & 1.12 \\
\hline 7 & $\begin{array}{l}\text { Mulch } 4 \text { t/ha fb Bispyribac } \\
\text { fb One hw }\end{array}$ & 4513BCD & $5883 \mathrm{CD}$ & 1.34 & 1.82 & 0.34 & 0.82 \\
\hline 8 & $\begin{array}{l}\text { Stale seedbed fb mulch } 4 \\
\text { t/ha fb Bispyribac }\end{array}$ & $3485 \mathrm{E}$ & $4719 \mathrm{~F}$ & 1.07 & 1.55 & 0.07 & 0.55 \\
\hline 9 & $\begin{array}{l}\text { Pendimethalin and Sesbania } \\
\text { co-culture fb2,4-D Na salt fb } \\
\text { one hw }\end{array}$ & $4385 \mathrm{CD}$ & $5671 \mathrm{D}$ & 1.35 & 2.02 & 0.35 & 1.02 \\
\hline 10 & $\begin{array}{l}\text { Pendimethalin } \mathrm{fb} 2,4-\mathrm{D} \mathrm{fb} \\
\text { one hw }\end{array}$ & $5161 \mathrm{AB}$ & $6351 \mathrm{~A}$ & 1.77 & 2.22 & 0.77 & 1.22 \\
\hline & Mean & 4181.65 & 5440.55 & & & & \\
\hline & $\mathrm{CV} \%$ & 10.86 & 3.66 & & & & \\
\hline & F-test & $* *$ & $* *$ & & & & \\
\hline & LSD at 0.05 & 659 & 290.2 & & & & \\
\hline
\end{tabular}

Note. $\mathrm{fb}=$ followed by $\& \mathrm{hw}=$ hand weeding 


\section{References}

Ampong-nyarko, K and SK Dc Datta. 1991. A Handbook for Weed Control in Rice. International Rice Research Institute, Los Banos, Philippines, p.113.

Belder P, BAM Bouman, JHJ Spiertz, Peng S, AR Castaneda and RM Visperas. 2005. Crop performance, nitrogen and water use in flooded and aerobic rice. Plant soil 273. 167-182.

De Datta SK and AM Beltazar. 1996. Weed control technology as a component of rice production systems. In. Auld, B.A; Kim, K.U.(Eds.), Weed management in Rice FAO plant production and protection paper 139,FAO, Rome. pp 27-52.

Elliot PC, DC Navarez, DB Estario and K Moody. 1984. Determining suitable weed control practices for dry-seeded rice. Philipp. J. Weed Sci. 11,70-82.

Fujisaka S, K Moody and Ingram K 1993. A descriptive study of farming practices for dry seeded rainfed lowland rice in India, Indonesia and Myanmar. Agric. Ecosyst. Environ. 45,115128.

Giri, GS, 1988. Effects of rice and wheat establishment techniques on wheat grain yield. In. Hobbs, P.R; Bhandari, R. (Eds.). Proceedings of Rice-wheat Research End of Project Workshop, India,pp. 65-68.

Hobbs PR, Y Singh, GS Giri, JG Lauran and JM Duxbery. 2002. Direct-seeding and reduced tillage options in the rice-wheat systems of the Indo-gangetic plains of South Asia. In. Pandey, S; Mortimer, M; Wade, L; Tuong, T.P; Hardy, B. (Eds.). Direct-seeding in Asian Rice systems. Strategic Research Issues and Opportunities. International Rice Research Institute, Philippines, pp.201-215.

Ladha, JK, D Dawe, H Pathak, AT Padre, RL Yadav, B Singh, Y Singh, P Singh, AL Kundu, R akal, N Ram, AP Regmi, SK Gami, L Bhandari, R Amin, CR Yadav, EM Bhattarai, S Das, HP Agrawal, RK Gupta and PR Hobbs. 2003. How extensive are yield declines in long term rice-wheat experiments in Asia. Field crops Res. 81, 159-180.

MOAC. 2009. Statistical Information Nepalese Agriculture, Agri-Business, Promotion and Statistics Division, Ministry of Agriculture

Moody K. 1991. Weed management in rice. In pimental, D (Ed). Hand book of pest management in agriculture, $2^{\text {nd }}$ Ed. CRC Press, Boca Raton. FL. USA, pp 301-328.

Ramamoorthy, K, A Arokiaraj and A Balasubramanian. 1998. Effect of irrigation and chemical weed control on crop yield and nutrient uptake by upland rice and associated weeds under rice-blackgram intercropping system. Oryza 33, 264-268

Ranjit, JD, KP Bhurer, KP Koirala, Y Thakur and DN Chaudhary. 1989. Screening of herbicides in upland and transplanted rice. pp. 129-139. In proc. $14^{\text {th }}$ summer crops workshop, Parwanipur, Nepal.

Rao AN, DE Johnsson, B Siva Prasad, JK Ladha and AM Mortimer. 2007. Weed management in direct-seeded rice. Adv. Agron.93, 153-255.

Rodder W. 2001. Slash and burn rice systems in the hills of Northern Lao PDR. In. Description, challenges and opportunities IRRI, LOS Banos, Philippines, 201 pp. 
Singh, S, L Bhusan, KK Ladha, RK Gupta, AN Rao, and B Sivprasad. 2005. Weed management in dry seeded rice (Oryza sativa) cultivated in the furrow-irrigated raised-bed planting system. www. elsevier.com/locate/cropo.

Singh, S, L Bhusan, KK Ladha, RK Gupta, AN Rao, and B Sivprasad, 2006. Weed management in Dry-seeded rice (Oryza Sativa) cultivated on furrow irrigated raised bed Planting System. Crop Prot. 25, 487 - 495.

Singh, YG, VP Singh, P Singh, RSI Shrivastava, A Saxena, M Mortimer, DE Johnson, and JL White. 2002. Effect of different establishment methods on rice-wheat and the implication of weed management in Indo-Gangetic Plains. In. proceedings of the International workshop on Herbicide Resistance management and zero tillage in Rice-wheat cropping system, 4-6 March 2002. Department of Agronomy, CCS Hryana Agricultural University, Hisar, India, pp.188-192.

Tuong TP and BAM Bouman. 2003. Rice production in water scare environments. In. Kijne, J.W; Barker, R; Molden, D. (Eds.). Water productivity in Agriculture. Limits and Opportunities for improvement. CABI Publishing, pp. 53-67.

Valverde BE and J Gressel. 2005. Implication and containment of gene flow from herbicideresistant rice (Oryza sativa). In "Proceeding of $20^{\text {th }}$ Asian Pacific weed sciences society" pp 63-84. Ho Chi Minh City, Vietnan. 